MDMA or 'Ecstasy' is widely used and popularly regarded as safe

- Systemic effects may include sudden death.

- Excessive thirst is quenched by the consumption of sugary carbonated drinks.

- Tooth surface loss and TMJ symptoms may result from tooth clenching and grinding

\title{
Ecstasy related periodontitis and mucosal ulceration - a case report
}

\author{
W. J. Brazier, ${ }^{1}$ D. K. Dhariwal, ${ }^{2}$ D. W. Patton, ${ }^{3}$ and K. Bishop, ${ }^{4}$
}

Methylenedioxymethamphetamine (MDMA) more commonly known as 'Ecstasy' is a widely used recreational drug. The oral and systemic effects associated with its use have been well documented. This paper highlights a previously unreported complication of MDMA use on the oral mucosa. MDMA periodontitis is illustrated with a case report and the local oral and systemic effects of MDMA use outlined.

Methylenedioxymethamphetamine (MDMA) is a 3,4-methylenedioxy ring substituted amphetamine which is more commonly known by several names such as 'XTC', 'E' and 'Love Drug.' ${ }^{1}$ MDMA was first synthesized in 1914 for use in chemical warfare, however it was not used therapeutically until the early 1970s when psychologists used it as a facilitating agent for psychotherapy. ${ }^{2}$ It is now a class A listed drug under the 1971 Misuse of Drugs Act. The illegal use of MDMA has become widespread in Britain, popularised in the early 1990's at 'raves'. It is a sympathomimetic stimulant which is taken orally, or occasionally injected intravenously, and causes a sense of euphoria, insomnia and suppresses appetite. It is relatively easy to obtain and fashionably regarded as a 'safe' drug despite serious medical sequelae including death. Decriminalisation of

\footnotetext{
${ }^{1}$ Senior House Officer in Oral and Maxillofacial Surgery, ${ }^{2}$ Specialist Registrar in Oral and Maxillofacial Surgery,

${ }^{3}$ Consultant in Oral and Maxillofacial Surgery, ${ }^{4}$ Consultant in Restorative Dentistry and Implantology, Maxillofacial Unit, Morriston Hospital, Swansea, SA6 6NL, Wales Correspondence and requests for offprints to: Miss D K Dhariwal, Maxillofacial Unit, Morriston Hospital, Swansea SA6 6NL, Wales

E-mail: daljitdhariwal@hotmail.com
}

\section{Refereed Paper}

Received 13.05.02; Accepted 24.10.02

๑ British Dental Journal 2003; 194: 197-199
MDMA and other less addictive drugs such as Cannabis has been considered.

The systemic effects associated with the use of MDMA have been well documented and are summarised in Table 1. Acute toxicity effects are not dose related nor in the majority of cases are they caused by impurities in the tablets. ${ }^{3,4}$ Metabolism occurs

by demethylation primarily in the liver and brain, catalysed by cytochrome $\mathrm{p} 450,{ }^{5}$ although up to $75 \%$ of the drug is eliminated unchanged via urinary excretion. ${ }^{2}$

\section{Oral implications}

The interactions of 'Ecstasy' with monoamine oxidase inhibitors and tricyclic anti-

\section{Table 1 Systemic effects associated with the use of MDMA}

\section{Psychiatric}

Agitation, delirium, panic disorder, flashbacks, paranoia, hallucination, depression, psychosis and suicidal ideation.

\section{Neurological}

Ataxia, seizures, tremor, tics, nystagmus and cerebral haemorrhage

\section{Cardio-vascular}

Tachyarrythmias, hypertension, myocardial ischaemia / infarction, and ventricular ectopy

\section{Respiratory}

Pulmonary hypertension and oedema

Hepatorenal

Renal failure, urinary retention, myoglobinuria, jaundice, hepatitis and hepatic failure necessitating liver transplantation ${ }^{15}$

\section{Metabolic}

Metabolic acidosis, hyponatraemia, hypoglycaemia, rhabdomyolysis, hyperthermia

\section{Gastrointestinal}

Nausea, vomiting, anorexia, diarrhoea, ischaemic colitis

Other

Disseminated intravascular coagulation (DIC), vasculitis, sexual dysfunction, seretonin syndrome, aplastic anaemia and death. 
depressants, which may be prescribed in the management of TMJ pain and atypical facial pain, resemble a tyramine reaction. Selective serotonin reuptake inhibitor (SSRI) antidepressants are competitive inhibitors of MDMA hepatic metabolism at the debrisoquine hydroxylase (CYP2D6) enzyme stage $^{5}$ and may potentiate the acute adverse effects of Ecstasy.

Drug abusers show a tolerance to local anaesthetics and conscious sedation and may require greater quantities of anaesthetic agent to achieve pain free dental treatment. ${ }^{1}$

The oral effects of MDMA are a manifestation of the systemic effects of the drug. Documented oral manifestations of MDMA use are summarised in Table 2. Dry mouth, bruxism and jaw clenching are common acute effects of MDMA use. The thirst may be quenched by the consumption of sugary carbonated drinks leading to extensive cervical caries and erosion. Thirst may also be quenched by excessive consumption of water and self-induced water intoxication is recognised in patients with ecstasy poisoning. The Ecstasy induced trip may last up to 36 hours such that tooth clenching and grinding is prolonged. The bruxism and clenching may lead to muscle tenderness and stiffness and coupled with the excessive consumption of acidic beverages, tooth surface loss (TSL) is compounded. ${ }^{6-8}$

\begin{tabular}{l} 
Table 2 Oral manifestations of MDMA use \\
\hline - Bruxism \\
- Tooth clenching \\
- TMJ arthromyalgia \\
- Dental attrition \\
- Xerostomia \\
- Mucosal ulceration \\
- Periodontitis
\end{tabular}

This paper describes a complication of MDMA use apparently not described previously, occurring as the result of topical application of the drug.

\section{CASE REPORT}

A 15-year-old boy was referred to the Oral and Maxillofacial Surgery Unit by his general medical practitioner with a one day history of general malaise and pyrexia and a painful upper anterior lip swelling. There had been no history of trauma. The patient had completed 14 months of fixed appliance orthodontic treatment 4 months previously, and had a well maintained dentition with good oral hygiene. There were no pathological periodontal pockets, ie pocket depths of 2-3 mm were noted.

Clinical examination revealed a swelling of the maxillary labial vestibule in

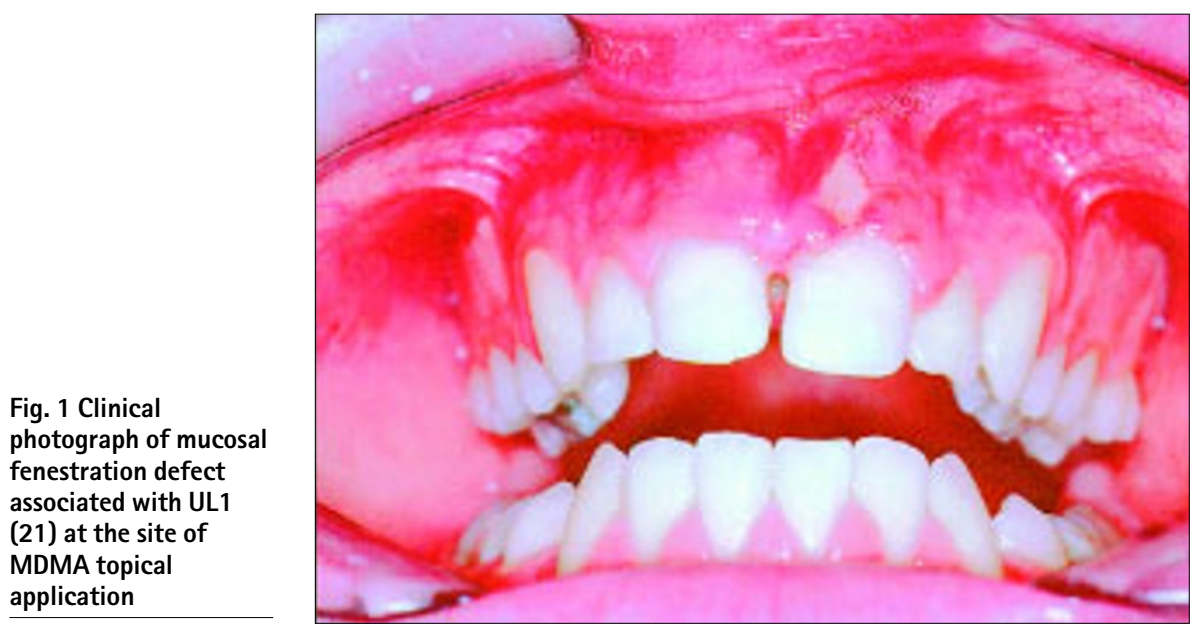

relation to the upper central incisors. Both maxillary central incisors exhibited Grade II mobility and were tender to percussion, but were otherwise sound. Incision and drainage of the presumed acute dentoalveolar abscess was performed and yielded serous fluid which was sent for culture. Routine blood tests were performed. Oral antibiotics, Penicillin V and Metronidazole were commenced.

Laboratory studies revealed a white cell count (WCC) of $8.2 \times 10^{9} / 1$ [normal range 5-10 × $10^{9} / 1$ ], predominantly eosinophils. The erythrocyte sedimentation rate (ESR) was elevated at $23 \mathrm{~mm} / \mathrm{hr}$ [normal range 2-10 $\mathrm{mm} / \mathrm{hr}$ ] and hepatic enzymes aspartate transaminase (AST) and gamma glutamyl transferase (GGT) elevated at 59U/1 and $43 \mathrm{U} / 1$ respectively [normal range AST $=0-40 \mathrm{U} / \mathrm{l} ; \mathrm{GGT}=0-85 \mathrm{U} / \mathrm{l}$. Microbiological culture yielded viridans streptococci, staphylococcus epidermidis and corynebacterium species, consistent with commensal oral flora.

On review 2 days later the swelling had reduced in size and systemic symptoms subsided. Mucosal fenestration of the attached gingiva with exposure of the underlying alveolar bone and roots of the UL1 (21) and UL2 (22) was noted with Grade II mobility of UR2 (12), UR1 (11), UL1 (21) and UL2 (22) (Fig. 1). The teeth were all vital on electric and thermal pulp testing. Radiographic examination confirmed no significant loss of bony support (Fig. 2).

Detailed questioning in the presence of his parents revealed no additional information. The patient denied using any topical agents in the area.

He was referred to the dental hygienist for oral hygiene instruction, scaling and polishing. At this visit he reported that he had used 'Ecstasy' as a recreational drug one day prior to onset of symptoms and stored the drug in the upper anterior labial vestibule adjacent to the site of periodontal destruction. The patient denied use of other recreational drugs and previous use of Ecstasy. The diagno-

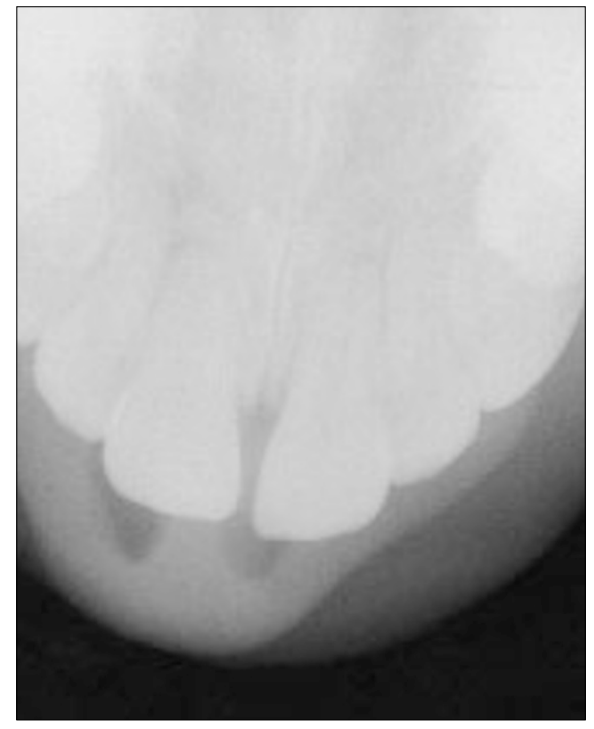

Fig. 2 Upper anterior occlusal radiograph shows evidence of mild blunting of the roots of the teeth but no significant bone loss

sis of local drug induced necrotising gingivitis was made.

Cessation of the use of MDMA was advised, and oral hygiene education provided. Sub-gingival debridement and supragingival scaling of the involved teeth was performed. Fluoride [Duraphat 2.26\% Sodium fluoride 5\% varnish; Colgate] was also applied to the exposed roots to relieve dentinal sensitivity. An orthodontic retainer was constructed to support the mobile upper anterior teeth. The mucosal ulcer had healed at 1 week review and 4 months after presentation the teeth were firm and vital. The patient at this time had no complaints, although a $2-3 \mathrm{~mm}$ asymptomatic localised defect of the alveolar gingivae and supporting bone remained, and he was discharged back to his general dental practitioner for regular periodontal maintenance.

\section{DISCUSSION}

MDMA is widely accepted within the general population as a relatively safe drug and is becoming more frequently used, 
despite its potentially serious side effects. These are not related to the dose or frequency of use. The malaise and fatigue reported in this case is said to occur within hours or days of MDMA use. ${ }^{9}$ The blood biochemistry profile with mildly elevated enzymes confirms a hepatitic picture consistent with MDMA use. The time interval between drug use and onset of symptoms is consistent with acute effects of MDMA.

There have been no previous reports of oral mucosal or periodontal lesions associated with abuse of MDMA, although similar lesions to those seen in this case have been reported with the local application of cocaine. These include gingival and mucosal ulceration and periodontitis ${ }^{10-12}$ which occur following chronic gingival application. This is thought to occur as a result of the ischaemic vasoconstricting action of the drug. ${ }^{12,13}$ Cervical abrasions and gingival lacerations have also been described and are thought to be the result of excessive toothbrushing during a 'high.'14 This patient reported that he stored MDMA in the upper vestibule. This was unusual as the drug is usually ingested orally immediately, unlike cocaine which when rubbed into the oral mucosa is well absorbed.

The labial mucosa adjacent to the area of drug application was not affected. This might be due to :

- Different mucosal types - the firmly attached alveolar mucosa versus loosely attached labial mucosa such that prolonged contact with one area of epithelium may not occur on the lip

- Exposure to salivary flow - minor salivary gland flow from the upper lip might wash away chemical irritants better than alveolar mucosa

- Method of ecstasy application - if the tablet were held onto the alveolar mucosa by the tip of the tongue whose epithelium is more keratinised, this might not be affected and would shield the lip from the presumed chemical burn.

Parry et al. ${ }^{10}$ reported a case of a 14-year-old multiple drug abuser who applied cocaine and amphetamines onto the upper labial vestibule and presented with a clinical picture suggestive of necrotising gingivitis, with eythema and ulceration of the gingiva adjacent to the site of drug application. This was thought to be a local drug reaction caused by cocaine. However, the patient had also used MDMA in the same way but local effects of this drug were not considered. Juvenile periodontitis in an immunosuppressed patient can present with a similar clinical picture but this explanation did not coincide with the clinical findings in this patient.

This case report highlights a previously unreported effect of MDMA abuse which may present to the general dental practitioner. It is increasingly likely that dentists will encounter patients who abuse these recreational drugs. This paper highlights the need for a high index of suspicion of drug abuse on presentation of unusual periodontal conditions.

1. Rees T D. Oral effects of drug use. Crit Rev Oral Biol \& Med 1992; 3: 163-184.

2. Shannon M. Methylenedioxymethamphetamine (MDMA, 'Ecstasy') Paed Emer Care 2000; 16: 377-380.

3. Wolff K, Hay A W M, Sherlock K et al. Contents of 'Ecstasy'. Lancet 1995; 346: 1100-1101.

4. Henry J A, Jeffreys K J, Dawling S. Toxicity and deaths from 3,4-methylenedioxymethamphetamine, ("Ecstasy"). Lancet 1992; 340: 384-387.

5. Burgess C, O'Donohoe A, Gill M. Agony and ecstasy: a review of MDMA effects and toxicity. Eur Psychiatry 2000; 15: 287-294.

6. Millosevic A, Agrawal N, Redfearn PJ et al. The occurrence of toothwear in users of Ecstasy $(3,4$ MethyleneDioxyMethAmphetamine). Community Dent Oral Epidemiol 1999; 27: 283-287.

7. Murray M C, Wilson N H F. Ecstasy related tooth wear. BrDentJ 1998; 185: 264.

8. Duxbury A J. Ecstasy - Dental implications. Br Dent J 1993; 175: 38.

9. McCann U D, Slate S O, Ricaurte G A. Adverse reactions with 3,4-methylenedioxymethamphetmine (MDMA: 'Ecstsy'). Drug Saf 1996; 15: 107-115.

10. Parry J, Porter S, Scully C et al. Mucosal lesions due to oral cocaine use. Br Dent J 1996; 180: 462-464.

11. Lee CYS, Mohammadi H, Dixon R A. Medical and dental implications of cocaine abuse. J Oral Maxillofac Surg 1991; 49: 290-293.

12. Yukna R A. Cocaine periodontitis. Int J Periodont Restor Dent 1991; 11: 73-79.

13. Gargiulo AV, Toto P D, Gargiulo A W. Cocaine induced gingival necrosis. Periodontal Case Rep 1985; 7: 44-45.

14. Friedlander A H, Gorelick D A. Dental management of the cocaine addict. Oral Surg, Oral Med, Oral Path 1988; 65: 45-48.

15. Garbino J, Henry J A, Mentha G et al. Ecstasy ingestion and fulminant hepatic failure: liver transplantation to be considered a last therapeutic option. Vet Human Toxicol 2001; 43: 99-102. 